\title{
Revisitando o Conceito de Mecanismo de Coordenação: Confiança Como Um Mecanismo de Coordenação na Análise de Formas Organizacionais Plurais no Agronegócio
}

\author{
Reviewing the Concept of Coordination Mechanism: Trust as a Coordination Mech- \\ anism in the Analysis of Plural Organizational Forms in Agribusiness
}

\begin{abstract}
Resumo
A questão de coordenação, de alocação de recursos e das formas organizacionais utilizados para esta alocação, tem sido destacado como um foco de investigação em diferentes ciências e abordagens teóricas, tais como economia, sociologia e Teoria Organizacional, entre outros. Embora os modelos, estruturas de governança e formas organizacionais tenham merecido grande destaque, o mesmo não ocorre com o conceito de mecanismo de coordenação, evidenciando a necessidade de revisão e reconstrução deste conceito. Confiança, considerada por muitos autores como sendo um dos mecanismos de coordenação, tem sido utilizada como uma forma complementar e integrada com outras abordagens. No agronegócio, mesmo que seja mencionado, são poucas as obras que a contemple de forma mais profunda. A confiança foi escolhida para este ensaio como um esforço para elucidar algumas questões teóricas relativas ao mecanismo de coordenação. Assim, o objetivo deste trabalho é revisitar de forma aprofundada o conceito de mecanismo de coordenação. Conclui-se que a confiança atende aos propósitos de um mecanismo de coordenação, e que é necessário uma melhor identificação das arenas e mecanismos de coordenação e as possiveis combinações de mecanismos na identificação de formas organizacionais.
\end{abstract}

Palavras-chave: Confiança, mecanismo de coordenação, dependência. Interdependência, interdependencies, governança.

\begin{abstract}
The matter of coordination, of allocation of resources and of the organizational forms used for this allocation, has been highlighted as an investigative focus in different sciences and theoretical approaches such as economics, sociology, and Organizational Theory, amongst others. Although models, governance structures and organizational forms have deserved great prominence, the same doesn't occur with the concept of coordination mechanism, showing the necessity of reviewing and rebuilding of this concept. Trust, considered by many authors as being one of the mechanisms of coordination, has been used as a complementary and integrates form to other approaches. In agribusiness, even though it's mentioned, few are the works that contemplates them in a more deep way. It has been chosen make this essay as an effort to elucidate the theoretical matters approached. Thus, the goal of this work is reviewing the concept of coordination mechanism. It is concluded that trust attends to the proposals of a coordination mechanism and that it's necessary the wellidentification of the arenas and coordination mechanisms and the possible combinations of mechanisms in the identification of organizational forms.
\end{abstract}

Key words: trust, mechanism of coordination, dependencies, interdependencies, governance.

\section{Recebido: 01/12/2014 Aceito: 15/04/2015}

Wilson Magela Gonçalves ${ }^{1}$ e Tania Nunes da Silva²

${ }^{1}$ Universidade Federal de Lavras, Doutorado em Agronegócios - magela@ dag.ufla.br

${ }^{2}$ Universidade Federal do Rio Grande do Sul, Doutorado em Sociologia - tnsilva@ea.ufrgs.br 


\section{Introdução}

A questão da coordenação, da alocação dos recursos e das formas organizacionais utilizadas para esta alocação, tem-se destacado como foco investigativo em diferentes ciências e abordagens teóricas tais como a economia, sociologia, teoria organizacional, dentre outras. Três formas organizacionais, ou estruturas de governança, com seus respectivos mecanismos de coordenação têm sido mencionadas por estes estudos. Mercado, hierarquia e formas híbridas. Preço e autoridade têm sido consenso como mecanismos de coordenação das formas organizacionais mercado e hierárquica, respectivamente. No entanto, a forma organizacional Híbrida, não possui o mesmo consenso. Vários mecanismos têm sido indicados. As formas híbridas apresentam uma diversidade de tipologias, com características distintas uma das outras. Contratos de longo prazo, co-produção, comércio recíproco, contratos de distribuição, alianças estratégicas, joint-ventures, franquias, redes de suprimento, dentre outros, são alguns exemplos que podem ser citados.

As formas híbridas são vistas como promissoras em função de poderem proporcionar o equilíbrio entre a competição e cooperação; reduzirem os custos, inclusive os de transação; no longo prazo orientam relacionamentos, permitem uma mútua flexibilização e o uso comum de know-how técnico e econômico. Ressalta-se, porém, que a importância destas formas organizacionais não pode ser reduzida apenas à questão da redução dos custos de transação. A forma de organização do tipo híbrida envolve contratos complexos e arranjos de propriedade parcial de ativos que, por sua vez, criam uma relação bilateral entre as partes que pode ser caracterizada por dependência ou interdependência.

Qual seria o mecanismo de coordenação das formas hibridas? A literatura aponta para uma diversidade de possíveis mecanismos. Mas, talvez a questão mais importante seja a forma que estes mecanismos são utilizados na definição das formas organizacionais, se exclusiva (trade off) ou de forma combinada.

Enquanto modelos, estruturas de governança, formas organizacionais têm merecido um grande destaque, o mesmo não acontece com o conceito de mecanismo de coordenação. Revisitar, reconstruir, o conceito de mecanismo de coordenação é impor- tante neste momento por várias razões. Uma delas é que a literatura internacional e nacional menciona exaustivamente este termo. Contudo, pouco se tem discutido a cerca do que é realmente um mecanismo de coordenação, e de sua amplitude analítica. Em geral associado a esta concepção temos as estruturas e formas organizacionais. Mas, o que pode ser considerado como um mecanismo de coordenação? Para responder a esta pergunta é necessário caracterizar o que é coordenação, bem como o que é um mecanismo.

Nos estudos do agronegócio a utilização dos conceitos de coordenação e de formas organizacionais tem também recebido atenção dos pesquisadores. Com uma forte influencia da Economia dos Custos de Transação. Mas, paulatinamente tem recebido contribuições das outras áreas do conhecimento, principalmente da sociologia e da teoria organizacional. Estudos multidisciplinares têm sido realizados, procurando entender melhor os fenômenos ocorridos nesta área.

A confiança, considerada por muitos autores como sendo um dos mecanismos de coordenação, tem sido utilizada de forma integrada ou complementar a outras abordagens. No agronegócio, mesmo mencionada, poucos são os trabalhos que contemplam a confiança de uma forma mais aprofundada. Compreender como a presença ou ausência da confiança influencia nas formas organizacionais é de crucial importância. Optou-se por realizar este ensaio como um esforço de elucidar as questões teóricas a serem abordadas em tese de doutorado.

Assim, o objetivo deste trabalho é revisitar o conceito de mecanismo de coordenação, no sentido de verificar a condição do uso da confiança como mecanismo de coordenação. Para isto desenvolvese uma proposta de conceito de mecanismo de coordenação, em seguida sistematizam-se as relações de dependência e interdependência, as arenas, os mecanismos e suas correspondentes formas organizacionais, incluindo as possíveis combinações de mais de um mecanismo. Na seção três, apresentamse algumas considerações a cerca da coordenação no agronegócio. Na seção quatro apresenta-se uma revisão sobre confiança na tentativa de vislumbrar os elementos que ratifique ou não a afirmativa de alguns autores como sendo um dos mecanismos de coordenação para que possa ser utilizado nos estudos em agronegócio. Na seção cinco, apresentam-se as considerações finais sobre o tema, e por fim têm- 
se as referências que serviram de base para esse estudo.

\section{Revisitando o conceito de mecanismo de coordenação}

O conceito de mecanismo de coordenação é amplamente citado e assumido pelos diversos autores como um conceito dado. Nos trabalhos internacionais encontraram-se poucos autores que discutem coordenação (Thompson, 1967) ; Malone e Crowston, 1994) e menos ainda que discutem a questão do mecanismo. Que características possuem os mecanismos de coordenação para serem considerados como tal? Por que preço e autoridade podem ser considerados como mecanismo? Existem outros mecanismos?

A definição de mecanismo de acordo com o dicionário Aurélio é: combinação de peças, de órgãos, para fazer funcionar, para pôr em movimento um aparelho, um engenho, dando o sentido de maquinismo. A derivação por extensão de sentido é um conjunto de elementos que concorrem para a atividade, para o funcionamento, de uma estrutura orgânica, agora com sentido de mecânica. Mecanismo cerebral e fisiológico são alguns exemplos. Ainda no sentido figurado seria a maneira de operar comparável à de uma máquina, de uma engrenagem, sendo os mecanismos da política e os mecanismos que comandam uma empresa alguns dos exemplos.

$\mathrm{Na}$ economia, mecanismo é: "In economics a mechanisms a set of rules designed to bring about a certain outcome through the interaction of a number of agents each of whom maximizes his or her own utility".

Desdobrando nos seus elementos constituintes o conceito pode ser mais bem visualizado.

- Set of rules = a noção de conjunto é a essência do conceito de mecanismo. Conjunto de (rules), normas, procedimentos, regras, padrões;

- designed to bring about a certain outcomes =A definição de rules, em um dos seus sinônimos (sociologia) pode ser utilizada no sentido de indução a um determinado padrão de comportamento. Isto é um resultado. Pode se discutir com base na literatura qual seria o resultado esperado.

- throught the interaction of number of agents = Através da das interações entre agentes. É necessário caracterizar estas interações.
- each of whom maximizes own utility $=\mathrm{O}$ que as pessoas e as organizações buscam quando participam de um processo interativo? Superficialmente falando elas buscam a complementaridade de objetivos. Oriundo da teoria neoclássica, este conceito merece ser aprofundado no sentido de identificar a maximização da utilidade.

$O$ sentido de mecanismo reforça-se quando acrescido da definição de coordenação. Etimologicamente co-ordenar vem do latim "Ordinatione" quer dizer dispor segundo certa ordem, organizar, arranjar. E, o prefixo "co" significa com ou em conjunto. Assim, coordenação seria ordenar em conjunto ou realizar um trabalho ordenado coletivamente. Esta definição também é ratificada por Furlanetto e Arbage (2000), onde afirmam:

$\mathrm{Na}$ verdade, a expressão "coordinator", em inglês tem um sentido de "ordenador" ou "harmonizador", bem diferente de sua tradução para o português - coordenador. Portanto, ao utilizarmos as expressões coordenador e coordenação, nós as estamos fazendo no sentido original e dado por Coase (1937), ou seja, de "ordenador" e de "ordenação" das atividades produtivas respectivamente.

A chave fundamental neste processo passaa ser então definir além de o que é coordenação, e que é coordenável. Malonee Crowston (1994), discutindo a questão da interdisciplinaridade da coordenação, iniciam suas considerações dizendo que: A key insight of the framework presented here is that coordination can be seen as the process of managing dependencies among activities.

$\mathrm{O}$ argumento central destes autores é que se não existe interdependência não existe a necessidade de coordenação, e este argumento é consistente com os argumentos de diversas teorias. Este conceito também pode ser desdobrado nos seus elementos constituintes:

- managing $=$ administrar, gerir, ordenar, harmonizar as atividades realizadas pelas organizações (firmas) e entre as firmas.

- dependencies among activities= Segundo Mintzberg e Quinn (2001), toda atividade humana organizada da margem a dois requisitos fundamentais e opostos, a divisão do trabalho em várias tarefas e a coordenação destas tarefas. Isto da margem a pensar nas tarefas, ou seja, nas atividades, na sequência destas tarefas, na interdependência das mesmas. Como será visto mais adiante, a dependência e a 
interdependência podem ser em diferentes níveis e terem denominações também diferentes.

Feitas estas observações pode-se então operacionalizar o seguinte conceito para mecanismo de coordenação: Mecanismo de coordenação é um conjunto de princípios, regras, normas, condutas, padrões, processos com o propósito de obter determinados resultados através da (interação) administração das dependências entre atividades de um número de agentes onde cada qual procura realizar a melhor adaptação diante das mudanças provocadas pelo ambiente.

$\mathrm{O}$ aspecto central do conceito pode ser entendido como sendo a administração das dependências e interdependências entre as partes. Explorar as dependências é um enfoque diferente de outros trabalhos que valorizam as tipologias de formas organizacionais.

\subsection{Relações de Dependência e Interdependência}

O que deve ser levado em consideração para chegar aos mecanismos? Como os agentes, organizações se relacionam? Um dos autores mais mencionados quando se discute coordenação e relações de dependência é Thompson (1967). Outros autores utilizam de sua taxonomia no sentido de apresentar uma correlação entre tipos de interdependência, mecanismos de coordenação e formas organizacionais. Mesmo sendo voltada para as organizações em especifico, a taxonomia foi estendida para relações resultantes das interações entre organizações, redes, cadeias, por exemplo, (BORYS e JEMISON, 1989). Um dos autores que utiliza a taxonomia de Thompson de forma mais diretamente é Henry Mintzberg.

Segundo Mintzberg e Quinn (2001), toda atividade humana organizada dá margem a dois requisitos fundamentais e opostos, a divisão do trabalho em várias tarefas e a coordenação destas tarefas, consequentemente deve-se pensar nas tarefas, na sequência destas tarefas, na interdependência das mesmas. Thompson (1967) aponta 03 tipos de interdependência, pooled interdependence, sequential interdependence e reciprocal interdependence $\mathrm{e}$ afirma que "Numa situação de interdependência, a ação conjunta sucede através da coordenação; e se há diferentes tipos de interdependência, esperamos encontrar diferentes artifícios para alcançar a coordenação.”Os artifícios são entendidos por alguns autores com sendo mecanismos de coordenação
(MINTZBERG，1995; MINTZBERG e QUINN, 2001).

Numa perspectiva multidisciplinar, Sheppard e Sherman (1998), apresentam outra tipologia quando se trata da questão de dependência e interdependência. Partindo de Fiske (1990) que apresenta 04 tipos de formas relacionais: communal sharing, authority ranking, equality matching e marketing pricing, eles acrescentam a perspectiva da profundidade do relacionamento (Deep e Shalow) e os riscos associados a cada uma destas. O resultado então passa a ser 02 tipos básicos de dependência (Shalow e deep) e 02 tipos de interdependência (Shalow e deep). Cada uma destes tipos possui riscos associados, que poderão ser mitigados com cada tipo de qualidade de confiabilidade. Segundo Sheppard e Sherman (1998) existe uma relação entre os tipos de interdepencia pooled e sequentialde Thompson (1967) e a shalow depedence.

Nesta altura do cabem alguns questionamentos. Coordenação e controle têm o mesmo significado? O que pode ser controlado e coordenado? Com base em Mintzberg (1995), assume-se neste trabalho que coordenação relaciona-se com controle, ... "A coordenação, contudo, provou ser uma questão mais complicada, fazendo apelo a vários meios. Estes podem ser referidos como "mecanismos de coordenação", embora se deva notar que esses mecanismos têm tanto a ver com o controlo e a comunicação como com a coordenação." O autor ainda menciona que conforme alguns autores, como Litterer (1965), por exemplo, controle e coordenação têm a mesma essência.

Admitindo que controle e coordenação tenham a mesma essência podemos trabalhar com os conceitos de controle de Ruigrok e Von Tulder (1995), onde os autores relacionam controle, barganha, dependência e interdependência e tipologias de networks para o controle (coordenação). Admitem que nos processos produtivos (produção artesanal /especialização flexível, fordismo e toyotismo), uma firma utiliza-se de diversas maneiras para controlar cinco áreas vitais: o processo de trabalho, suprimento de componentes e matéria prima, distribuição e consumo, tecnologias centrais de produção e finanças. Numa perspectiva de barganha, pode-se destacar duas relações de dependências compatíveis com as apresentadas por outras abordagens teóricas até o momento, relação entre uma organização e seus fornecedores e com seus compradores. Sendo as- 
sim, pode-se entender que o sentido de controle dado aqui pelos autores pode ser o mesmo de coordenação.

Malone e Crowston (1994) apresentando uma perspectiva multidisciplinar para a coordenação, identificam como elemento central desta coordenação, conforme já foi visto anteriormente, "...coordination can be seen as the process of managing dependencies among activities". Decorrente desta afirmação eles passam a identificar quais seriam as "...dependencies among activities", para correlacionar com cada mecanismo de coordenação. Segundo estes autores algumas destas dependências seriam: recursos compartilhados, relacionamentos de produtores e consumidores, tarefas/subtarefas, etc. Porém, eles lembram que não é uma lista fechada de dependências e que é necessário desenvolve-la cada vez mais. Além da coordenação os autores defendem que "... Similarly, even though words like "cooperation," "collaboration," and "competition" each have their own connotations, an important part of each of them involves managing dependencies between activities"

\subsection{As Arenas e os Mecanismos de Coordenação}

Uma importante contribuição a respeito de mecanismos de coordenação e formas organizacionais é feita por Imaie Itami (1999). Inicialmente eles definem que existem as arenas onde os recursos devem ser alocados. Estas arenas são o mercado e a organização. Defendem também a existência de princípios ou mecanismos pelos quais os recursos são alocados, neste caso existem princípios utilizados pelas organizações e princípios utilizados pelo mercado. As combinações resultantes são: Mercado puro, organização pura, mercado que funcionam como organizações, organizações que funcionam como mercados e organizações intermediárias. A questão da combinação de mecanismos de coordenação é fundamental para o avanço do entendimento das formas organizacionais resultantes, posição esta defendida por outros autores, como será visto adiante. Caracterizar as principais arenas e os mecanismos utilizados pelas mesmas e suas combinações é imprescindível. Portanto a partir deste ponto utilizaremos a distinção de arena e mecanismos (princípios). Isto significa entender onde e como ocorrem as relações de dependência.
O mecanismo de coordenação da arena mercado é preço, existe pouca ou nenhuma divergência com relação a este fato. Da mesma forma acontece com o mecanismo de coordenação autoridade correspondente à arena organização. No caso de autoridade o que existe, conforme já foi demonstrado Thompson(1967) e Mintzberg (1995), é uma variedade de modos de se praticar a autoridade. No entanto existe uma diversidade de possibilidades de formas organizacionais do tipo híbrida, e mecanismos de coordenação correspondentes. Um dos mecanismos citados por diversas abordagens teóricas é a confiança.

O conceito de coordenação, dentro da perspectiva econômica, teve Coase (1937) como um dos pioneiros. Ele considerava que: "Outside the firm, price movement direct production which is coordinated throughta series of exchange transactions on the market. Within a firm, these markets transactions are eliminated and in place of the complicated market structure with exchange transactions is substituted the entrepreneur co-ordinator, who directs production. It is clear that these are alternative methods of co-ordinating production." A noção de ordenação das atividades produtivas (coordenação, conforme visto acima) é estabelecida. Ainda percebe-se nitidamente a arena organização e mercado como espaços onde ocorre a coordenação, aparecendo o empreendedor como o coordenador da produção.

Depois de Coase (1937), Thompson (1967), na perspectiva da teoria organizacional, tratou da questão da coordenação, associando-a com interdependência e mecanismos para a sua obtenção. Ele indicou 03 maneiras de se alcançar a coordenação dentro das organizações:

- padronização, de planos e ajuste mútuo e menciona que “... há paralelos distintos entre os três tipos de interdependência e os três tipos de coordenação. Com a interdependência reunida, a coordenação por padronização é apropriada; com a interdependência sequencial, é apropriada a coordenação por plano; e com a interdependência recíproca, aplica-se a coordenação por ajuste recíproco." (THOMPSON, 1967). Outra questão importante apontada pelo autor refere-se à questão dos desdobramentos em termos de comunicação e decisão, ou seja, “... os três tipos de coordenação, na ordem apresentada acima, impõem fardos cada vez mais pesados às comunicações e decisões". É importante ressaltar este aspecto 
uma vez que alguns autores indicam a comunicação como um mecanismo de coordenação (GRANDORI e SODA, 1995).

Mintzberg (1995), aponta cinco mecanismos de coordenação e cinco configurações para as organizações. Porém Mintzberg e Quinn (2001) revisaram e acrescentaram mais um mecanismo que é a padronização de normas e duas configurações. Contudo, mais importante do que citá-los é entender que os trabalhos destes autores referem-se à forma organizacional-organização.

Nassimbeni (1998), utiliza os conceitos deThompson(1967) e Mintzberg (1995) para relacionar formas organizacionais (Main-contractor and subcontracting units, Production spinnerets, Agreements, Joint-Ventures, Regional industrial systems) com tipos de interdependência (de fluxo, escala, processo e processo), com o principal mecanismo de coordenação (supervisão direta, padronização de entradas e saídas, de habilidades e de processo), respectivamente.

Em termos de mecanismos de coordenação, de acordo com Queiroz (2004), pode-se considerar que Richardson (1972), seja uma ponte não intencional entre os trabalhos iniciais de Coase (1937), e os autores posteriores a ele, tais como Williansom(1991, 1993, 1996) e Ouchi (1985). Richardson (1972) percebeu que as empresas estruturavam-se em torno de atividades similares especializando-se ao invés de realizarem atividades complementares, que exigiriam a cooperação entre firmas para coordenar as atividades não similares.

Segundo este autor as atividades similares e complementares, de responsabilidade de diferentes firmas podem representar papéis diferentes nas cadeias produtivas, o que é similar para um pode ser complementar para outro.

Segundo Queiroz (2004), Richardson (1972) pode ser considerado o precursor das estruturas de governança williamsonianas, em função de destacar três alternativas para as quais as atividades de coordenação sejam efetivadas. Direção, equivalente á hierarquia, cooperação interfirmas, equivalente às híbridas; e, mercado de transações, equivalente ao próprio mercado.

Com base em Coase (1937) e Macneil (1978), os trabalhos apresentados pela Economia dos Custos de Transação (ECT), Williamson (1991, 1993, 1996), identificam três formas mecanismos de coordenação, preço, autoridade e contratos, corres- pondentes a três estruturas de governança: mercado, hierárquica (firma) e híbrida, respectivamente.

Ouchi (1980,1985), analisando a forma multidivisional, apresenta uma variante do modelo de configurações organizacionais de Williansom, classificando-os em mercado, burocracias e clãs econômicos. Ele considera que os clãs econômicos tiveram origem nos acordos sociais entre membros da comunidade e gerariam alternativas que explicariam as firmas da sociedade japonesa. Neste caso, tradição surge como um terceiro mecanismo de coordenação, alternativo ao preço e autoridade. Considerando as arenas e as combinações, Ouchi (1985) não vislumbra combinações de mecanismos, apresenta uma perspectiva de "trade-off', ou seja, na medida em que se usa a tradição(confiança), utiliza-se menos o preço e a autoridade.

Bladach e Eccles (1989), e Pihl (2001), separam formas de mecanismos de coordenação e defendem formas plurais. Estas formas plurais são resultado da combinação de diversos mecanismos. Enquanto os primeiros autores defendem preço, autoridade e confiança como mecanismos, Pihl (2001) defende preço, autoridade, regras e ideologia (confiança e socialização).

Zenger e Hesterly (1997) afirmam que existe uma tendência de um "inchaço" da zona entre o mercado e hierarquia, neste caso contrariamente a Williansom (1991), as formas híbridas seriam uma espécie de amálgamaentre mercado e hierarquia. $\mathrm{O}$ ponto de proliferação das formas híbridas seria onde ocorresse alto incentivo de poder dentro das firmas e controle hierárquico dentro dos mercados.

Adler (2001) introduz uma concepção quese diferencia das concepções que consideram os mecanismos de forma isolada. O elemento central da sua proposta é a inserção da confiança. Isso representa um avanço quando se trata das posições de Imai e Itami (1999). Na visão deste autor, existem três dimensões que podem ser combinadas. Os vetores são: comunidade/confiança, mercado/preço e hieraquia/autoridade. $\mathrm{Na}$ perspectiva tridimensional as combinações resultantes seriam: "spot market", contratos relacionais, Burocracia representativa, Burocracia Coercitiva, Formas Híbridas de alta confiança, Formas Híbridas de baixa confiança, Clã, Horda associal.

Esses trabalhos acima mencionados evidenciam a necessidade já mencionada de se identificar claramente as arenas e os princípios (mecanismos de 
coordenação), bem como a necessidade de se utilizar combinações de diversos mecanismos na identificação das formas organizacionais.

\section{A utilização do Conceito de Coordenação no Agronegócio}

Tanto a ECT quanto outras abordagens teóricas têm discutido o que é coordenação. As formas organizacionais (formas de governança) são decorrentes de como dá a questão dos incentivos, controles administrativos, adaptação e tipos de contratos (WILLIAMSON, 1991). Muitas vezes o conceito de coordenação vem acompanhando da noção de cadeia produtiva, onde se procura identificar quais as formais organizacionais predominantes. Segundo Arbage (2004), existe uma vinculação entre coordenação, cadeias produtivas e os aspectos da eficiência e desempenho. Batalha e Scramim (1999) consideram que uma melhor coordenação entre os agentes diminui os custos incidentes sobre cada um deles, os conflitos são menores e a adaptação ao ambiente também será melhor. Com a finalidade de reduzir custos de transação, os agentes fazem uso de mecanismos apropriados para regular uma determinada transação.

Pioneiros na discussão de coordenação no agronegócio no Brasil, Farina e Zylbersztajn (1994), dão um sentido operacional ao conceito onde coordenação que é “... a habilidade de transmitir informação, estímulos e controle ao longo das etapas sequências que integram o conjunto de atividades necessárias para atender o mercado". A arena é a cadeia produtiva. Os elementos identificados para a efetivação da coordenação “... transmitir informações, estímulos e controle..." podem e devem ser entendidos como exercício da autoridade, elaboração de planos, padronização e ajuste mútuo. Informa-se o que? Estimula-se o que? E controla-se o que?

Analisando o caso especifico das supply chain, e o conceito de das cadeias estritamente coordenadas Farina e Zylbersztajn(1999), partem do pressuposto que : (1) uma cadeia de suprimento pode ser encarada com um nexo de contratos ampliado, cuja arquitetura resulta do alinhamento com as características das transações e o ambiente institucional; (2) existem arranjos contratuais que reproduzem a arquitetura contratual no nível da firma e a motivação para a elaboração de um subsistema parte das estratégias de mercado e busca de eficiência em custos de transação, geralmente adotada por algum agente dentro da cadeia.

Trabalhos posteriores como os de Saes e Farina (1999) e Saes e Nakazone (2002), consideram que um subsistema estritamente coordenado ocorre quando um padrão de concorrência demanda a adoção de estruturas de governança específicas, caso dos produtores de café orgânico e da empresa Illycaffe. Os autores Azevedo e Faulin (2003), também definem cadeias estritamente coordenadas como "...o encadeamento de transações que se sucedem para produzir um produto final, em que partes e contrapartes são identificadas e asseguram a continuidade da relação por meio de uma estrutura de governança particular."

A arena apresentada pelos autores que discutem os subsistemas estritamente coordenados é a cadeia de suprimento que assume as características de uma firma (organização). Se isto acontece, os mesmos mecanismos de coordenação indicados para uma organização podem ser indicados para a cadeia de suprimento. Esta noção é defendida por Lazzarini, Chaddad e Cook (2001), onde correlacionam os tipos de interdependência de Thompson (1967), pooled interdependence, sequential interdependence e reciprocal interdependence, com os mecanismos de coordenação planos, padronização e ajuste mútuo, respectivamente.

Arbage (2004), estudando estruturas hibridas de governança em empresas agroalimentares no Rio Grande do Sul, conceituou coordenação como sendo a "habilidade de transmitir informação, estabelecer medidas de orientação e assistência, conduzir estímulos e implementar medidas de controle ao longo das etapas sequências de produção, visando adequar os objetivos de eficiência da cadeia de suprimentos aos interesses do consumidor". Este autor identifica as "variáveis" que devem ser consideradas para compreender a formação de mecanismos de coordenação das ações organizacionais em um sistema agroindustrial, ou em uma cadeia produtiva em particular. Ele identifica sistemas de informação, medidas de incentivo e do exercício do controle, e inclui mais uma variável que é a assistência técnica.

Furlanetto e Zawislak (2000) desenvolveram um ensaio teórico onde apresentam o conceito de coordenação pela cadeia produtiva - a coordenação sistêmica. Que irá coordenar todas as atividades da cadeia, permitirá a diminuição dos custos de transa- 
ção (ECT), administrar as interdependências e promover uma cooperação Competitiva (Dependência de Recursos), união de diferentes elos em uma rede virtual(Redes de Empresas). Na verdade o que se apresenta é mais uma tipologia, coordenação sistêmica. O que está presente nesta conceituação é que menciona-se a interdependência, e os mecanismos de coordenação contratos, e confiança. Porém não fica evidente a arena e os princípios ou mecanismos de coordenação.

Pelos poucos trabalhos analisados, e em função da natureza deste ensaio, percebe-se que os trabalhos a respeito de coordenação no agronegócio precisam avançar no sentido de se introduzir variáveis relacionais, a exemplo da confiança. Além disso, é necessário analisar as relações de dependência para identificar melhor os mecanismos de coordenação. As tipologias das diversas formas organizacionais, principalmente as hibridas, são importantes como modelos de abstração, para entender a realidade, mas são consequências das relações e dos mecanismos.

\section{Confiança Dimensão Quase Esquecida ou Não Descoberta Pelos Estudos do Agronegó- cio}

A confiança tem sido contemplada por diversas abordagens teóricas, psicologia, psicologia social, filosofia, sociologia, economia, teoria das organizações, administração estratégica. Tem sido utilizada de forma integrada ou complementar a outras abordagens. A literatura sobre o tema é vasta e reflete esta diversidade, alguns trabalhos sintetizam esta amplitude. Com base em alguns trabalhos procurarse-á identificar elementos que credencie ou não a confiança como mecanismo de coordenação. Para isto é preciso analisar a confiança como elemento que propicie a adaptação, incentivo ou controle no processo de relacionamentos interpessoais ou interorganizacionais.

Tschannen-Moran e Hoy (2000), numa perspectiva multidisciplinar analisam a natureza, significado e mensuração da confiança. Apresenta uma longa lista de autores com seus respectivos trabalhos e conceitos desconfiança. São 14 conceitos organizados cronologicamente, começando com dois conceitos clássicos, o de Deutsch (1958), onde o mesmo refere-se à confiança como uma expectativa de comportamento, onde o individuo num determinado evento percebe consequências negativas e positivas na ausência ou presença de confiança e o de Rotter, (1967), onde a confiança interpessoal é uma expectativa mantida por um individuo ou grupo de que a palavra, promessa verbal ou escrita de outro individuo ou grupo pode ser confiável.

Das e Teng (2004) apresentam outro framework conceitual sobre a visão baseada no risco da confiança. Este trabalho também apresenta uma grande variedade de conceitos de confiança oriundos de diversas abordagens teóricas. Alguns conceitos são coincidentes com os apresentados por TschannenMoran e Hoy (2000), outros foram acrescidos.

Além dos conceitos, sistematizam os principais tipos, dimensões e componentes da confiança. A contribuição dos autores é classificar os conceitos, tipos, dimensões e componentes em quatro categorias: antecedentes de confiança; confiança subjetiva (goodwill trust, competence trust), confiança comportamental; e confiança relacionada com o risco, para depois combiná-los no sentido de sustentar a visão baseada no risco da confiança. Mas, além da sistematização, Das e Teng (2004) concluem que: confiança não é uma sub-classe de risco, na realidade, eles são a imagem de espelho de um ao outro; a categoria de confiança subjetiva, apresentada pelos autores, engloba os tipos de confiança goodwill ecompetence de Barber, (1983), onde a percepção de risco inclui risco relacional e risco de performance.

Outro trabalho bastante citado na literatura sobre confiança é o de Mayer e Davis et al., (1995). A primeira grande contribuição dos autores é no sentido de diferenciar confiança (trust) de cooperação, confidence epredictability. Embora a confiança possa frequentemente conduzir à cooperação, a confiança não é necessariamente condição para que ocorra a cooperação. $\mathrm{O}$ fator utilizado para tal diferenciação é o risco. A circunstância de cooperação não coloca necessariamente qualquer uma das partes em risco. Os autores citam como exemplo as situações de trabalhos interdependentes onde o empregado é "obrigado" a cooperar para a realização da tarefa.

Balestro e Mesquita (2002), discutindo a questão da confiança nas relações interorganizacionais apontam para a importância da confiança afirmando que em níveis mais elevados de cooperação ela pode contribuir para a formação de estratégias coletivas, facilitar a coordenação das atividades econômicas, promover a troca de informações e a aprendi- 
zagem interorganizacional, e contribuir também para a redução dos custos de transação. Como considerações finais apontam uma importante questão para a utilização da confiança. “... é importante realçar que o tema da confiança além de possuir implicações bastante relevantes para estudos sobre a cooperação e arranjos interorganizacionais, necessita ser examinado com maior profundidade sob várias facetas. Isto porque a confiança não é apenas plural nas suas definições, mas nos diferentes contextos da sua existência e construção". Eles afirmam, também, que poucos foram os trabalhos empíricos encontrados sobre confiança.

A confiança, também, é classificada de diversas maneiras pelos autores, sendo que a de Lane (1998), e Child e Faulkner (1998) uma das mais citadas. São apontadas três perspectivas sobre as bases da confiança; a confiança calculista ou calculativa, a confiança cognitiva e a confiança normativa. A confiança calculativa, segundo Lane (1998), "trusting involves expectations about another, based on calculations which weight the cost and benefits of certain courses of action to either the trustor or the trustee." Esta perspectiva de confiança baseia-se na visão do homem como ator racional, sendo racionalidade no sentido utilitarista e maximizadora. Este tipo de confiança, segundo Lewicki e Bunker (1996), é baseado na garantia de que as partes agirão conforme o combinado, pois a punição por violar é maior que os possíveis ganhos, ou o contrário a recompensa por preservar é maior que a perda por quebrá-la. Importante assinalar que este tipo de confiança depende enormemente da disponibilidade de informações. Na prática, a presença da racionalidade limitada interfere significativamente nesta disponibilidade. Outra característica importante da confiança calculista é o fato dela se aplicar particularmente às relações que são novas, baseando-se, então, na proteção institucionalizada ou na reputação do parceiro.

A confiança do tipo cognitiva, denominadas por Lewicki e Bunker (1996) de confiança baseada no conhecimento (Knowledge-basedtrust), é baseada na previsibilidade do outro, sustentando-se pela informação em lugar do impedimento dela. Aqui a confiança é fundamentada na confiança e no conforto deque o parceiro é bem compreendido e é conhecido a ponto de compartilhar importantes suposições. Só é possível uma das partes compartilhar o pensamento do outro através de um maior conheci- mento do mesmo (LANE, 1998 ; CHILD e FAULKNER, 1998).

A terceira visão de confiança é chamada normativa, que depende do compartilhamento de valores comuns entre as pessoas, inclusive deum conceito comum de obrigação moral. De acordo com Lane (1998), valores comuns e normas de obrigação podem se desenvolver se a relação existe há muito tempo. Segundo Lewicki e Bunker(1996), esta confiança existe porque as partes além de entender o desejo uma da outra, ainda apreciam estes desejos. Existe uma grande possibilidade da amizade se desenvolver ao longo do tempo, habilitando então uma pessoa a sentir e pensar como o outro.

\section{Considerações Finais}

Partindo do conceito desenvolvido, percebe-se que o aspecto central do conceito de coordenação pode ser entendido como sendo a administração das dependências entre as partes. Explorar as dependências e interdependências é um enfoque diferente de outros trabalhos que valorizam as tipologias de formas organizacionais.

Fica evidenciada a necessidade, de se identificar claramente as arenas e os princípios (mecanismos de coordenação), bem como a necessidade de se observar as possíveis combinações de diversos mecanismos na identificação das formas organizacionais.

Pelos poucos trabalhos analisados, e em função da natureza deste ensaio, percebe-se que os trabalhos a respeito de coordenação no agronegócio precisam avançar no sentido de introduzir variáveis relacionais, a exemplo da confiança. Além disso, é necessário aprofundar na análise das relações de dependência no sentido de identificar melhor os mecanismos de coordenação. As tipologias das diversas formas organizacionais, principalmente as hibridas, são importantes como modelos de abstração, para entender a realidade, mas são consequências das relações e dos mecanismos.

Percebe-se que a confiança mencionada na seção anterior, atende aos propósitos de um mecanismo de coordenação, pois presta-se a administração das relações entre as partes, no sentido de uma melhor adaptação, de incentivar comportamentos e de propiciar algum tipo de controle. 


\section{Referências}

ADLER, P. S. Market, hierarchy, and trust: The knowledge economy and the future of capitalism. Organization Science, v.12, n.2, Mar-Apr, p.215-234. 2001.

ARBAGE, A. P. . Custos de Transação e a Gestão da Cadeia de Suprimentos: Estudos de Caso em Estruturas de Governança Híbridas do Sistema Agroalimentar no Rio Grande do Sul.In: XXVIII EnANPAD - Encontro da Associação Nacional de Pós-Graduação e Pesquisa em Administração, 2004, Curitiba-PR. XXVIII EnANPAD. Rio de Janeiro-RJ : ANPAD, 2004.

AZEVEDO, P. F. ; FAULIN, E. J. Subsistemas Estritamente Coordenados Baseados em Confiança: o caso de verduras.In: IV INTERNATIONAL CONFERENCE ON ECONOMICS AND AGRI-FOOD NETWORKSMANAGEMENT, 2003, Ribeiraão Preto-SP. IV INTERNATIONAL CONFERENCE ON ECONOMICS AND AGRI-FOOD NETWORKS MANAGEMENT. Ribeirão Preto_SP : PENSA, 2003. v. 1. p. 1-12.

BALESTRO, M.V. E MESQUITA, Z.Confiança nas relações interorganizacionais: aproximando conceitos, ensaiando reflexões. In: $26^{\circ}$ Encontro Nacional dos Programas de Pós-Graduação em Administração, 2002, Salvador. Anais. Rio de Janeiro: ANPAD, 2002. BATALHA, M.O.; SCRAMIM, F.C.L. Supply Chain Management em Cadeias Agroindutriais: Discussões Acerca das Aplicações no Setor Lácteo Brasileiro.II Workshop Brasileiro de Gestão de Sistemas Agroalimentares. PENSA/FEA/USP, Ribeirão Preto, 1999.

BRADACH, J. L. ; ECCLES, R. G. Price, authority, and trust: From ideal types to plural forms. Annual Review of Sociology, v.15, p.97-118. 1989.

BORYS, Br e JEMISON, D. B. Hybrid Arrangements as Strategic Alliances: Theoretical issues in organizational combinations. Academy of Management Review. Vol. 14, n 2.1989.

CHILD, J. ; FAULKNER, D. Strategies of cooperation: Managing alliances, networks and joint ventures. Oxford: Oxford University Press. 1998.
COASE, R.H. The Nature of the Firm. Econômica, n.4, nov. 1937

DAS, T. K. ; B. S. TENG. The risk-based view of trust: A conceptual framework. Journal Of Business And Psychology, v.19, n.1, p.85-116. 2004.

DEUTSCH, M. Trust and suspicion. Journal of Conflict Resolution, v.2, n.4, p.265-279.1958. FARINA, M.M.Q.; ZYLBERSZTAJN, D. Competitividade e Organização das Cadeias Agroindustriais. IICA - Instituto Interamericano de Cooperação para a Agricultura - Costa Rica, 1994.

FARINA, E. M. M. Q. ; ZYLBERSZTAJN, D. Strictly Coordinated Food-Systems: Exploring the Limits of the Coasian Firm. International Food And Agribusiness Management Review,Estados Unidos, v. 1, p. 249-265, 1999.

Fiske, A. P. Relativity within Moose (Mossi) Culture - 4 Incommensurable Models for Social Relationships. Ethos, v.18, n.2, Jun, p.180-204. 1990.

FURLANETO, E. ZAWISLAK, P.A. Coordenação pela cadeia produtiva: uma alternativa ao mercado e à hierarquia. In: Encontro da ANPAD EnAMPAD, 2000, Florianopólis. Anais....XXIV Encontro da ANPAD - EnAMPAD, 2000.

GRANDORI, A. ; SODA, G. Inter-firm network: antecedents, mechanisms and forms. Organization Studies, p. 183-214, 1995.

IMAI, K. E ITAMI, H. Interpenetration of organization and market: Japan's firm and market in comparison with the US. In: BUCKLEY, P.J.e MICHIE, J.(orgs.) Firms organizations and contracts. Oxford: Oxford University Press, 1999.

LANE, C. Introduction: theories and issues inthe study of trust. In: LANE, Christel, BACHMANN, Reinhard (orgs.) Trust within and between organizations. Oxford: Oxford University Press, 1998.

LAZZARINI, S. G.; CHADDAD, F.R., COOK, M. L. Integrating supply chain and network analysis: the study of netchains. Journal of Chain and Network Science, v. 1, n. 1, p. 13-22, 2001. 
LEWICKI, R. J. ; BUNKER, B. B. Developing and maintaining trust in work relationships. In: KRAMER, R. M., TYLER, T. R. (eds.) Trust in organizations. Thousand Oaks:Sage, 1996.

LITTERER, J.A. The analysis of organizations, Wiley, 1965.

MACNEIL, I. 1978. "Contracts: Adjustments of Long-Term Economic Relations Under Classical, Neoclassical, and Relational Contract Law", Northwestern University Law Review,72: 854906.

MALONE, T. W. E CROWSTON, K. The Interdisciplinary Study of Coordination. ACM Computing Surveys, 26(1), 87-119.1994

MAYER, R.C., DAVIS, J. H. ; SCHOORMAN, F.D. An Integrative Model of Organizational Trust. Academy of Management Review, v. 20, n. 3, p.709-734, 1995.

MINTZBERG, H.; QUIN, J. B. O processo da estratégia. 3. ed. Porto Alegre: Bookman, 2001.

MINTZBERG, H. Estrutura e Dinâmica das Organizações, Lisboa: Publicações Dom Quixote, 1995.

OUCHI, W. G. "Markets, Bureaucracies, and Clans." Administrative Science Quarterly, v.25, p.124-14, 1980.

OUCHI, William. Sociedade M: a Força do Trabalho em Equipe. São Paulo: Nobel, 1985.

PIHL, H. Price, authority, ideology and rules:Coordination mechanisms and co-ordination forms. Working Paper Series, v.2,p.1-16. 2001.

QUEIROZ , N. dos S. A Ciência da Organização Pós-Setenta à Luz das Vertentes Institucionalistas da Sociologia e da Economia. Tese (Doutorado em Administração) - Universidade Federal da Bahia. Bahia, 2003.

ROTTER, J. B. A new scale for the measurement of interpersonal trust. Journal of Personality, v.35, p.651-665. 1967.
RICHARDSON, G. B. The organization of industry, The Economic journal, Oxford/UK, v. 82, 883-96, 1972

RUIGROK, W. e VAN TULDER, R. The Logic of International Restructuring. The Management of Dependencies inRival Industrial Complexes. London : Routledge. 1995.

SAES, M. S. M.; FARINA, E. M. M. Q. O agribusiness do café no Brasil.1999. 230 p. SAES, M. S. M.; NAKAZONE, D. Estudos da competitividade de cadeias integradas: impactos das zonas de livrecomércio. São Paulo: FECAM/FIPE, out. 2002.

SHEPPARD, B. H. ; SHERMAN, D. M. The grammars of trust: A model and general implications. Academy of Management Review, v.23, p.422-437.1998.

THOMPSON, J. D. Organizations in Action, McGraw-Hill, Nova York, 1967.

TSCHANNEN-MORAN, M. ; W. K.Hoy. A multidisciplinary analysis of the nature, meaning, and measurement of trust. Review of Educational Research, v.70, n.4, Win, p.547-93. 2000.

WILLIANSOM, O.E. Comparative Economic Organization: The Analysis of Discrete Structural Alternatives. Administrative Science Quarterly, v. 36, n. 2, pp. 269-296, 1991.

Calculativeness, trust and economic organization'. The Journal of Law and Economics, v.36, n.1, p.453-486. 1993.

The Mechanisms of Governance. Oxford University Press, 429 p. 1996.

ZENGER, T.R ; HESTERLY, W.S. The disaggregation of corporation: selective intervention, high-powered incentives, and molecular units. Organization Science, v.8,n.3, p.209-222. 1997. 